\title{
Quantity perception: The forest and the trees
}

\author{
Sami R. Yousif \& Frank C. Keil \\ Yale University, Department of Psychology
}

Running Head : Quantity Perception: Forests and Trees

Addresses for : Sami Yousif

correspondence Department of Psychology

Yale University

Box 208205

New Haven, CT, 06520-8205

Email : sami.yousif@yale.edu

Word Count : 2100 (Main Text)

Version : Accepted - Cognition 


\begin{abstract}
Park (2021) has described "flawed stimulus design(s)" in our recent studies on area perception. Here, we briefly respond to those critiques. While the rigorous, computational approaches taken by Park (and others) certainly have value, we believe that our approach - one that focuses the perceptual reality of quantity rather than the physical reality — is essential. We emphasize again (as we have many times in our work) that the study of quantity perception benefits from both approaches. To further illustrate our point, we collected additional data and show that some of Park's arguments, while sensible in principle, further support our view in practice.
\end{abstract}


Consider the Ebbinghaus illusion, in which two equivalent discs - one surrounded by larger discs and one surrounded by smaller discs - appear different in size (see Figure 1). If we wanted to explain the Ebbinghaus illusion, we couldn't do it by measuring the central discs; they are identical. This illusion can be explained only by appeal to perception.

We have studied quantity perception with this idea in mind: That the perceptual reality may differ from the physical reality. (We made this same point about the Ebbinghaus illusion in the first paper we published on this topic; see Yousif \& Keil, 2019, p. 502; this point has also been made by others; see "Physical versus Perceptual Reality" in Aulet \& Lourenco, 2021, p. 2.) Our view is that we may be unable to understand area perception by exhaustively measuring every dimension in a display; sometimes, we must ask what is not being captured in the available measurements.

Park (2021) disagrees with us. He sees this approach as flawed. He argues that researchers should study quantity perception by using a predetermined set of orthogonalized dimensions. There is undoubtedly value in the rigorous, computational approaches taken by Park (and others; see, e.g., Castaldi et al. 2019; Cicchini et al., 2016; Van Rinsveld et al., 2020). These approaches have moved the field forward, and we have never questioned their merits. As we have regularly emphasized, "the field needs...both...approaches" (Yousif \& Keil, 2020, p. 9).

Different questions require different strategies. While we believe that the approach Park (2021) champions is useful for answering many questions about quantity perception, it may not be the most useful for answering our questions. Thus, we are tempted to say that Park is "not even wrong": It is just that one party is pointing at forests while the other is pointing at trees. Our modest proposal is this: We need to understand the forests and the trees.

That said, we want to briefly reply to a few specific issues raised by Park.

\section{Issue \#1: Is area perception veridical?}

Park frames our work in terms of two separate conclusions: (1) Area perception is not veridical, and (2) Area perception influences number perception. In response to the first conclusion, Park argues that our findings are merely a 'reiteration' of prior findings (p. 3) — as if taking it for granted that area perception is not veridical. The good news is that we agree with Park here: Area perception is illusory. (And we would add a corollary: Area perception is systematically illusory.) 
However, it does not seem possible to accept the first conclusion and reject the second. If area perception is illusory, then studies failing to account for this perceptual distortion must have intrinsic limitations. Just as Park highlights incongruities with our stimuli, one could highlight incongruities regarding perceived area in most prior studies. After all, most studies on quantity perception ignore the issue of area perception altogether, opting instead to manipulate only true, mathematical area. Manipulating true area is a sensible thing to do, of course unless true area does not map onto the perceptual reality. If area perception is systematically distorted, a natural question arises: How should we interpret number/area congruity effects (e.g., Barth, 2008; Hurewitz et al., 2006; Nys \& Content, 2012; Tomlinson et al., 2020; see discussion in Yousif \& Keil, 2021)? Surely, we must revisit them. This conclusion seems unavoidable, so long as we accept that area perception is illusory (as Park does). In other words: You can't have it both ways. Either area perception is distorted and we should study quantity perception with this in mind, or area perception is not distorted (at which point the many documented illusions of area perception demand an explanation).

\section{Issue \#2: Is 'additive area' a reflection of a flawed stimulus design?}

Park claims that the stimulus design in our study is flawed, and he offers several (useful) charts displaying bias in our stimuli. But flawed with respect to what? We agree that our stimuli were constructed in an unevenly distributed stimulus space (all studies ever conducted on quantity perception are, in some light). This is by design. Our real disagreement seems to be about whether/how these differences matter. We have three responses.

First, we purposefully used a wide range of approaches to stimulus creation across several papers, to ensure that our core findings are not specific to one task environment. Park characterizes this strategy as inconsistency on our part, while we see it as reflecting a conscious decision to opt for generalizability. 'Rigorous control' and 'generalizability' may seem to oppose one another in this research program - and that may explain how two equally well-intentioned researchers could come to quite different conclusions about how quantity perception ought to be studied.

Second, we must appeal to the principle of parsimony. Many of Park's claims are based on individual experiments, whereas we have consistently emphasized the need to interpret our findings collectively. When interpreted collectively, a parsimonious explanation of our findings 
is that 'additive area' explains area judgments. No other reasonable alternative has been proposed to account for the existing data.

Consider a few examples. First, Park argues that there is a bias with respect to number in some of our experiments. In other words, he is claiming that the effects we observe may be caused by confound with number that we failed to control. Although Park is correct that number is not tightly controlled in some experiments, number is purposefully manipulated in others. In some experiments, for example, we specifically orthogonalize number (see Experiment 4 of Yousif \& Keil, 2019; see also Experiments 1a and 1b of Yousif, Aslin, \& Keil, 2020). However, he argues that these findings should be dismissed entirely; they are not "directly subject to [his] main criticisms" (p. 8). But aren't they relevant? Park explains two different sets of our findings in two different ways: When number varies (e.g., Yousif \& Keil, 2019), number explains our findings related to area perception, but when number is controlled (e.g., Yousif, Aslin, \& Keil, 2020), apparently variance in dot sizes explains our findings. We prefer to stick to the one explanation that fits both data sets: additive area. Parsimony!

Here's a different example. In other studies, when we orthogonalize number with respect to our other dimensions of interest, not only does number fail to explain area perception, but it is inversely related to area perception in some cases (i.e., stimuli with more number are perceived as having less area; Yousif \& Keil, 2019). Park claims that this is evidence in support of his view. Number, he says, has a negative beta coefficient, but is still influencing area judgments. We find this confusing. Is Park arguing that some of our studies are explained by positive congruities between number and area, and others are being explained by negative congruities? As far as we know, only positive congruities between area and number have ever been documented (see, e.g., Barth, 2008; Hurewitz et al., 2006; Nys \& Content, 2012), and there is no theoretical account that predicts negative congruities. Does Park have any reason to predict when positive vs. negative congruities should occur? If not, then, once again: Parsimony!

We have emphasized repeatedly, in several different papers, that our data be interpreted collectively. We emphasize that point again here: There is never going to be a perfect experiment in any quantity perception study, ever. At best, a consensus might emerge across a range of studies. With this in mind, we don't get to decide which studies are and are not relevant; they are all relevant, unless we have compelling reasons to think otherwise. 
Third, Park argues that some of our effects (including the inverse congruity effect mentioned in the previous point) are explained by the "statistical regularities" of our stimuli across trials (e.g., p. 7) ${ }^{1}$. Fortunately, we need not wonder whether this is the case. This is a testable hypothesis. It is possible to have observers complete individual trials, without the context of the full stimulus set. If Park is correct, and our results are due in part to the statistics of our stimuli across trials, then our basic effects should not replicate. However, if our results instead reflect people's true impressions, then we should observe similar results, even when observers only see a single comparison.

We did exactly this ${ }^{2}$. We selected 16 stimulus pairs from critical trials of Experiment 4 of Yousif \& Keil (2019) and presented each of them to separate groups of 100 observers (original data can be seen in Figure 2A). Figure 2 shows two key findings. First, accuracy was higher overall in our replications. This makes sense for two reasons: (1) Observers in these replication samples had unlimited time to respond (unlike the original tasks), and (2) Observers only had to complete a single trial, and thus were less susceptible to fatigue effects. Second, there was a high correlation between accuracy in the original sample and in these new samples $(r=.86$; see Figure 2B). Thus, not only did we replicate the basic 'additive area' effect (i.e., blue/green bars are higher than yellow / red bars; see Figure 2C), we also demonstrated once again that (some) trials greater in number are perceived as having less area. Contrary to what Park implies, these effects cannot be explained by "statistical regularities" over time, because observers had no knowledge of the stimulus set from which each comparison was derived.

Suppose, however, that we are talking about a different kind of "statistical regularity". For example: Does it matter that our stimulus dimensions vary to different extents within a given trial? If additive area varies twice as much as number, we might think it is obvious that additive area would have a larger effect on responses than number (see, e.g., Cantrell \& Smith, 2013). Yet this possibility cannot explain the data we observe in practice. In the critical trials we replicated here, number is the dimension that varies most dramatically. Shouldn't this have made it less

\footnotetext{
${ }^{1}$ In reviewing this paper, Park claimed that we misconstrued this point, and that he was not referring to regularities over time but contained in the stimuli themselves. However, the paper we are responding to clearly refers to "regularities... over time" (p. 2). We are unsure what to make of this, so we have opted to respond to both arguments.

${ }^{2}$ In fact, such an experiment was already conducted via twitter — and our results replicate, even when observers weren't aware of the statistical regularities of our stimuli. As much as we'd have loved to let these twitter-poll data speak for themselves, we thought we ought to collect our own data.
} 
likely that we would observe effects of additive area (since number was the most salient dimension)? Moreover, the most extreme number ratios ( 1.60) are only slightly higher than the most extreme additive area ratios (1.25) — far from the 5:1 difference Park offered as an example. Additionally, many number ratios are actually lower than the corresponding area ratios. For all of these reasons, we think that additive area is the only viable, parsimonious explanation of the available data.

\section{Whack-a-mole}

We have presented several sets of studies documenting illusions of area perception. Park has raised several specific critiques that apply differently to each set of studies. In other cases, Park has refrained from commenting on studies that we have argued are relevant to his position. This strategy forces us to defend every single one of our dozens of experiments on different terms - resulting in what strikes us as an unwinnable game of Whack-a-Mole.

Instead of playing that game, we have repeatedly appealed to parsimony. Our goal was never to conduct a single bullet-proof experiment (no such experiment is possible), but to put forth a body of work that reveals a seemingly inescapable conclusion: Area perception is (systematically) illusory. As for how this influences quantity perception: We should revisit this question once studies have been conducted that take area perception seriously as a topic of interest unto itself. So far, little data meaningfully bear on this question. We appreciate Park's concerns about this, and we support an approach where his ideas, as well as ours, help guide future work.

We are not claiming we have found the one, true, perfect answer to area perception. There are certainly many open questions. But to answer those questions, the field needs a diverse set of perspectives - approaches that step back and approach the problem from different angles, and ones that address the vast space of intricate, computational details. Forests and trees. We hope that readers of our work (and of this dialogue) will step away appreciating the value in both strategies. 


\section{References}

Aulet, L. S., \& Lourenco, S. F. (2021). The relative salience of numerical and non-numerical dimensions shifts over development: A re-analysis of. Cognition, 210, 104610.

Barth, H. C. (2008). Judgments of discrete and continuous quantity: An illusory Stroop effect. Cognition, 109, 251-266.

Cantrell, L., \& Smith, L. B. (2013). Open questions and a proposal: A critical review of the evidence on infant numerical abilities. Cognition, 128, 331- 352.

https: / / doi.org/10.1016/j.cognition.2013.04.008

Castaldi, E., Piazza, M., Dehaene, S., Vignaud, A., \& Eger, E. (2019). Attentional amplification of neural codes for number independent of other quantities along the dorsal visual stream. ELife, 8. https: / / doi.org/10.7554/eLife.45160

Cicchini, G. M., Anobile, G., \& Burr, D. C. (2016). Spontaneous perception of numerosity in humans. Nature Communications, 7, 12536. https: / / doi.org/10.1038/ncomms12536

DeWind, N. K., Adams, G. K., Platt, M. L., \& Brannon, E. M. (2015). Modeling the approximate number system to quantify the contribution of visual stimulus features. Cognition, 142, 247265.

Hurewitz, F., Gelman, R., \& Schnitzer, B. (2006). Sometimes area counts more than number. Proceedings of the National Academy of Sciences, 103, 19599-19604.

Nys, J., \& Content, A. (2012). Judgement of discrete and continuous quantity in adults: Number counts! Quarterly Journal of Experimental Psychology, 65, 675-690.

Park, J. (2021). Flawed stimulus design in additive-area heuristic studies. Cognition, 104919.

Tomlinson, R. C., DeWind, N. K., \& Brannon, E. M. (2020). Number sense biases children's area judgments. Cognition, 204, 104352.

Van Rinsveld, A., Guillaume, M., Kohler, P. J., Schiltz, C., Gevers, W., \& Content, A. (2020). The neural signature of numerosity by separating numerical and continuous magnitude extraction in visual cortex with frequency-tagged EEG. Proceedings of the National Academy of Sciences, 117, 5726-5732. https: / / doi.org/10.1073/pnas.1917849117

Yousif, S. R., \& Keil, F. C. (2019). The additive-area heuristic: An efficient but illusory means of visual area approximation. Psychological Science, 30, 495-503.

Yousif, S., Aslin, R., \& Keil, F. (2020). Judgments of spatial extent are fundamentally illusory: 'Additive-area' provides the best explanation. Cognition. 205. 104439. 
Yousif, S. R., \& Keil, F. C. (2020). Area, not number, dominates estimates of visual quantities. Scientific Reports, 10, 1-13.

Yousif, S. R., \& Keil, F. C. (2021). How We See Area and Why It Matters. Trends in Cognitive Sciences. 


\section{Figures}
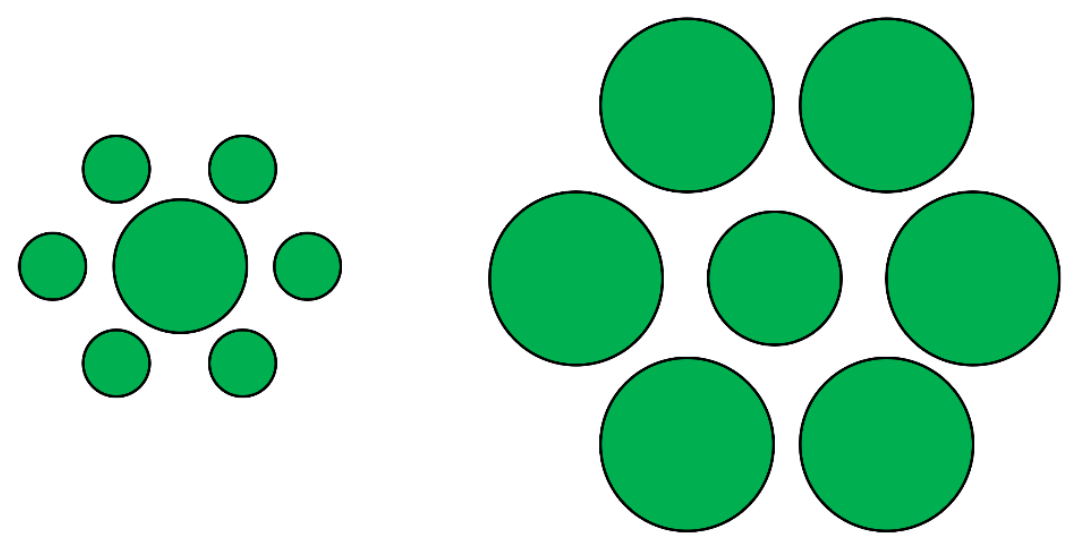

Figure 1. The Ebbinghaus illusion. 
A Original Data (Yousif \& Keil, 2019, Exp. 4)

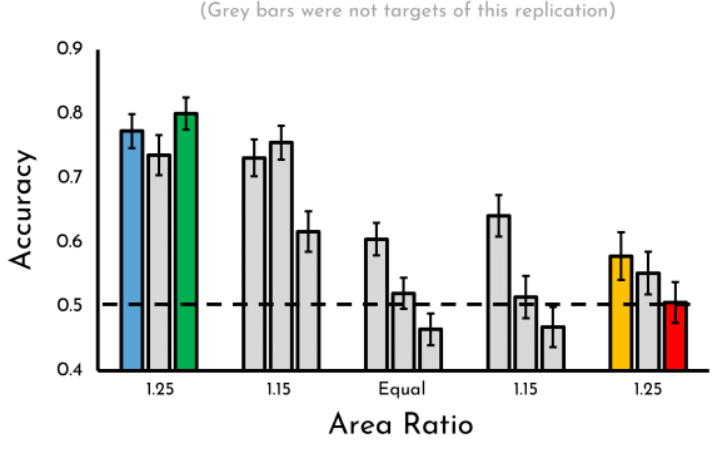

C Replication of critical trials

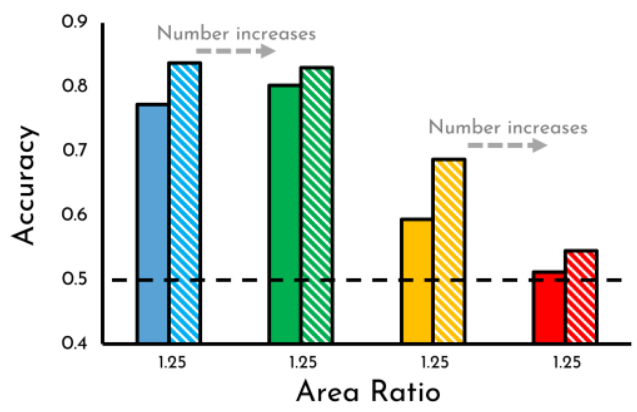

B

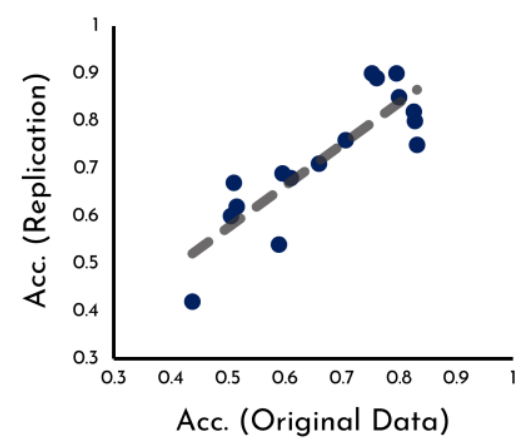

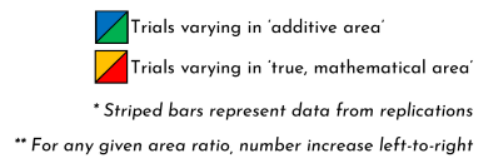

Figure 2. (A) Re-creation of results from Yousif \& Keil, 2019. Grey bars represent data that were not a target of this replication. Error bars represent + / - 1 SE. (B) Correlation between original data and the replication data. Accuracy across the sixteen stimuli was highly correlated between samples ( $r=.86)$. (C) Replication of critical trials from Yousif \& Keil, 2019 conducted as separate, one-shot experiments (i.e., observers only saw a single trial), compared side-by-side with the original data. Each bar represents four stimuli. Filled bars represent accuracy in the original sample. Striped bars represent accuracy in the replication sample. All relevant effects replicated. 